\title{
Molecular Characterisation and HIV Drug Resistance Patterns of HIV-1 Variants in Plasma and Peripheral Blood Mononuclear Cells Sample Pairs
}

\author{
Mzingwane $\mathrm{ML}^{1,2 *}$, Mayaphi $\mathrm{SH}^{1}$, Tiemessen $\mathrm{CT}^{3,4}$, Richter $\mathrm{K}^{1}$, Hunt $\mathrm{G}^{3}$ and Bowyer $\mathrm{SM}^{1}$
}

${ }^{1}$ Department of Medical Virology and National Health Laboratory Services TAD, University of Pretoria, South Africa ${ }^{2}$ Department of Pathology, National University of Science \& Technology, Bulawayo, Zimbabwe

${ }^{3}$ Centre for HIV and Sexually Transmitted Infections, National Institute of communicable diseases, Johannesburg, South Africa

${ }^{4}$ Faculty of Health Sciences, University of the Witwatersrand, Johannesburg, South Africa

\begin{abstract}
Objective: Plasma is currently the specimen of choice for routine HIV drug resistance (HIVDR) testing. However, HIV compartmentalization has been well documented in different reservoirs, including peripheral blood mononuclear cells (PBMCs). We molecularly characterized paired plasma and PBMC sample pairs and compared their HIVDR mutation patterns.
\end{abstract}

Methods: To compare HIVDR mutation patterns in plasma and PBMC sample pairs, whole blood was collected for plasma and PBMC isolation from 43 treatment-naïve and 10 treatment-experienced individuals, and HIVDR profiles determined by sequencing the $\mathrm{pol}$ gene. Treatment-naïve individuals were initiated on Efavirenz/Emtricitabine/ Tenofovir fixed dose combination therapy and follow up HIV-1 viral loads were performed after at least 6 months of treatment.

Results: HIVDR mutation prevalence in the treatment-naïve group was $5.1 \%$ in plasma samples compared to $10.3 \%$ when both plasma and PBMC sequences were considered. Variable amino acid positions were detected in $21 \%$ and $12 \%$ of the protease and reverse transcriptase genes, respectively. These were generally not in HIVDR positions and did not form signature patterns. The subset of patients with additional resistance associated sequence variations detected in PBMCs had undetectable HIV-1 viral loads after at least 6 months of anti-retroviral therapy.

Conclusions: The sequencing of paired plasma and PBMC specimens provided additional HIVDR data which were not detected when only plasma samples were tested. However the resistant variants detected in the PBMCs did not seem to negatively affect treatment outcome at 6 months as viral suppression was achieved. These data highlight proviral HIVDR mutations in HIV infected individuals and longer follow up on patients on treatment may be needed to determine their clinical impact.

Keywords: HIV drug resistance; HIV compartmentalization; PBMC reservoir

\section{Introduction}

With more than 35 million people currently infected with Human immunodeficiency virus (HIV) worldwide, the AIDS epidemic continues to be one of the greatest challenges of our time. In Sub Saharan Africa, home to more than two thirds of the infected population, the percentage of eligible people who are receiving highly active antiretroviral therapy (HAART) has risen from about 30\% in 2009 to above $60 \%$ in 2012 (UNAIDS report, 2012). HIV is characterized by high mutation rates and rapid evolution which results in creation of a wide variety of mutants that are present in an individual as viral quasi-species $[1,2]$. This allows the virus to be highly adaptable to new hosts and selection pressures. The virus is continuously changing to survive in new environments by selecting from this pool of pre-existing variants those variants with better fitness [3]. These variants may play a major role in viral evolution, virulence and evasion of the immune response and also compromise drug and vaccine development efforts. Since the introduction of HAART major successes in the fight against HIV have been achieved. The virus can now be suppressed to levels not detectable by standard viral detection and quantification molecular methods and successful treatment may fully block transmission [4]. However, the virus is only suppressed and not eradicated. Latent but replication-competent HIV genomes which persist in cellular and anatomical reservoirs may lead to treatment failure or viral rebound when treatment is interrupted [5].
Drug resistant variants play a major role in viral persistence and virological failure during HAART. HIV drug resistance testing is therefore a beneficial part of treatment optimization and individualized patient management. The choice of a regimen guided by the baseline resistance profiles would generally lead to improved outcomes compared to the absence of such resistance data and is recommended where feasible [6]. Full-length gene sequencing of the protease $(P R)$ and part of the reverse transcriptase $(R T)$ genes in the pol region of the HIV genome is done to determine whether any relevant mutations are present to provide a complete resistance assessment. Mutations in the genes encoding these enzymes underlie resistance to nucleoside/ nucleotide reverse transcriptase inhibitors (NRTIs), non-nucleoside reverse transcriptase inhibitors (NNRTIs), and protease inhibitors (PIs) which are the available drugs in the majority of developing countries. However HIV drug resistance testing is still not routinely carried out

*Corresponding author: Mzingwane ML, National University of Science and Technology, Department of Pathology, Faculty of Medicine, P. O Box AC939 Ascot, Bulawayo, Zimbabwe, Tel: +263 9 203336-9; E-mail: mayibongwe.mzingwane@nust.ac.zw

Received February 17, 2016; Accepted March 25, 2016; Published April 02, 2016

Citation: Mzingwane ML, Mayaphi SH, Tiemessen CT, Richter K, Hunt G, et al (2016) Molecular Characterisation and HIV Drug Resistance Patterns of HIV-1 Variants in Plasma and Peripheral Blood Mononuclear Cells Sample Pairs. J AIDS Clin Res 7: 562. doi:10.4172/2155-6113.1000562

Copyright: (C) 2016 Mzingwane ML, et al. This is an open-access article distributed under the terms of the Creative Commons Attribution License, which permits unrestricted use, distribution, and reproduction in any medium, provided the original author and source are credited. 
before treatment initiation and as part of treatment monitoring in developing countries due to limited resources.

Plasma is usually the specimen of choice for HIV resistance testing targeting circulating variants, but in light of virus compartmentalization in different reservoirs, antiretroviral resistance testing of viruses sequenced from plasma may not give a complete picture of an individual's resistance profile. Peripheral blood mononuclear cells (PBMCs) have been described as a latent viral reservoir containing productive HIV DNA [7]. Archived proviral DNA in PBMCs may also retain earlier forms of the virus which may sometimes re-emerge [1]. We characterized HIV variants in plasma and PBMC specimen pairs from treatment-naïve and treatment-experienced individuals and compared them for differences in resistance-associated mutations.

\section{Methods}

\section{Participants}

Participants were newly diagnosed HIV infected adults who were eligible for HAART initiation based on CD4+ count $(<350$ cells/ $\mu \mathrm{l})$ and/or World Health Organization (WHO) clinical staging and adults on HAART with symptoms of clinical failure and/or detectable viral load after at least one year on treatment. The participants were sequentially recruited from Tshwane District Hospital HIV clinic and F.F. Ribeiro Clinic in Pretoria Central, South Africa between July 2013 and May 2014 after written informed consent. Treatment-naïve individuals were initiated on a once per day Efavirenz/Emtricitabine/ Tenofovir fixed dose combination therapy and follow up HIV-1 viral loads were performed after at least 6 months of treatment. Ethics approval was obtained from the University of Pretoria Faculty of Health Sciences ethics committee (Protocol number 167/2013) and the Gauteng Province Tshwane Health Research Committee (Project number 25/2013).

\section{Sample collection and processing}

Plasma was isolated from $10-15 \mathrm{ml}$ of whole blood collected in EDTA tubes by centrifugation at $1600 \mathrm{~g}$ for 10 minutes and stored at $-70^{\circ} \mathrm{C}$ until required for RNA extraction. The volume of plasma isolated from whole blood was replaced with phosphate buffered saline (PBS) and PBMCs isolated on a one layer Ficoll Hypaque gradient. The PBMCs were washed three times in PBS and stored as dry pellets at $-70^{\circ} \mathrm{C}$ until DNA extraction.

\section{HIV genotyping and resistance testing}

RNA from plasma and proviral DNA from PBMCs, were extracted using QIAamp Viral RNA and DNA blood Mini kits (Qiagen) respectively as per manufacturer's protocols. Nucleic acid amplification and sequencing were done using previously described methods and primers [8]. Briefly, cDNA was produced from extracted RNA by use of Superscript III reverse transcriptase enzyme (Life Technologies Corporation, California, USA) and polymerase chain reactions (PCRs) were performed to amplify the protease gene and the first 300 codons of the RT gene (HXB2 nucleotide 2166 - 3440) using the Platinum Taq enzyme (Life Technologies Corporation, California, USA). PCR amplicons were visualized by ultraviolet illumination and the size validated against appropriate size markers after $1 \%$ agarose gel electrophoresis. Amplicons were cleaned and sequenced by BigDye Terminator V3.1 Cycle Sequencing Ready Reaction Kit (Applied Biosystems, Foster City, CA, USA) on a 3100 Automatic capillary sequencer (Applied Biosystems, Foster City, CA, USA). Sequencher V 4.5 (Gene Codes Corporation, USA) was used to edit and form contiguous sequences and the sequences were then submitted to the Stanford website (http://hivdb.stanford.edu/) for subtyping, resistance mutations interpretation and quality assessment using the Stanford HIVdb algorithm Version 7.0 [9]. Additional subtyping tools that were used included REGA V. 3 [10] (http://www. bioafrica.net/rega-genotype/html/subtypinghiv.html) and SCUEL [11] (http://www.datamonkey.org/dataupload_scueal.php). NCBI Basic Local Alignment Search Tool (Blast) analysis was used to confirm subtyping results (blast.ncbi.nlm.nih.gov/). For recombinant variants identification and analysis, bootscan analysis plots were constructed using REGA V. 3 [10] and s-distance similarity plots were constructed using the Recombinant Identification Program (RIP) V. 3 (http://www. hiv.lanl.gov/content/sequence/RIP/RIP.html).

\section{Phylogenetic analysis}

Reference sequences were obtained from the Los Alamos National Laboratory (LANL) database (http://www.hiv.lanl.gov/content/ sequence/NEWALIGN/align.html). Alignment was done using Clustal W [12] (Larkin et al., 2007) in BioEdit Sequence Alignment editor software (Tom Hall, North Carolina State University) and manually edited using BioEdit Sequence Alignment Editor. Maximum likelihood phylogenies were applied to determine evolutionary patterns and divergence rates using Mega 6 [13] with 1000 bootstrap values.

\section{Signature pattern analysis}

Viral Epidemiology Signature Pattern Analysis (VESPA) was used to search for PBMC associated signature patterns in the pol gene by determining the most common amino acid in each position compared to plasma derived HIV variants (www.hiv.lanl.gov/content/sequence/ VESPA/vespa.html). Analysis was done for amino acids in positions 1 - 99 in the $P R$ gene and positions $1-267$ in the $R T$ gene.

\section{Results}

\section{Participant characteristics}

A total of 53 participants were tested including 38 females and 11 males. Four additional sequences from samples obtained from the South African National Blood Service were included. The mean age of participants was 34 years (range $23-63$ years). Thirty nine participants were South African residents with no travel or stay history outside South Africa while 10 participants had travel or stay history in Botswana (1), Congo Brazzaville (1), Democratic Republic of Congo (1), Malawi (2), Mozambique (1) and Zimbabwe (4). There were 43 treatment naïve and 10 treatment experienced participants. Treatment naïve participants had a mean CD4 count of 210 (range 9 - 502).

\section{Phylogenetic analysis}

The sequences obtained are presented on the neighbor-joining phylogenetic tree in Figure 1 with bootstrap values of $70 \%$ and above indicated. Plasma and PBMC sequences from the same patient clustered together as expected. Fifty patients (94\%) were infected with HIV1 subtype $C$ strains, two were infected with circulating recombinant form 37 complex (CRF 37_cpx) and there was one unclassified subtype $\mathrm{C}$ related recombinant. The two sequences classified as CRF 37_cpx subtype came from epidemiologically and sexually related individuals and phylogenetically clustered. As shown in Table 1 both sequences were classified as CRF 37_cpx on the REGA platform but L074 was classified as PR: CRF01_AE, RT: G while L075 was classified as CRF02_ AG on the Stanford subtyping platform. 


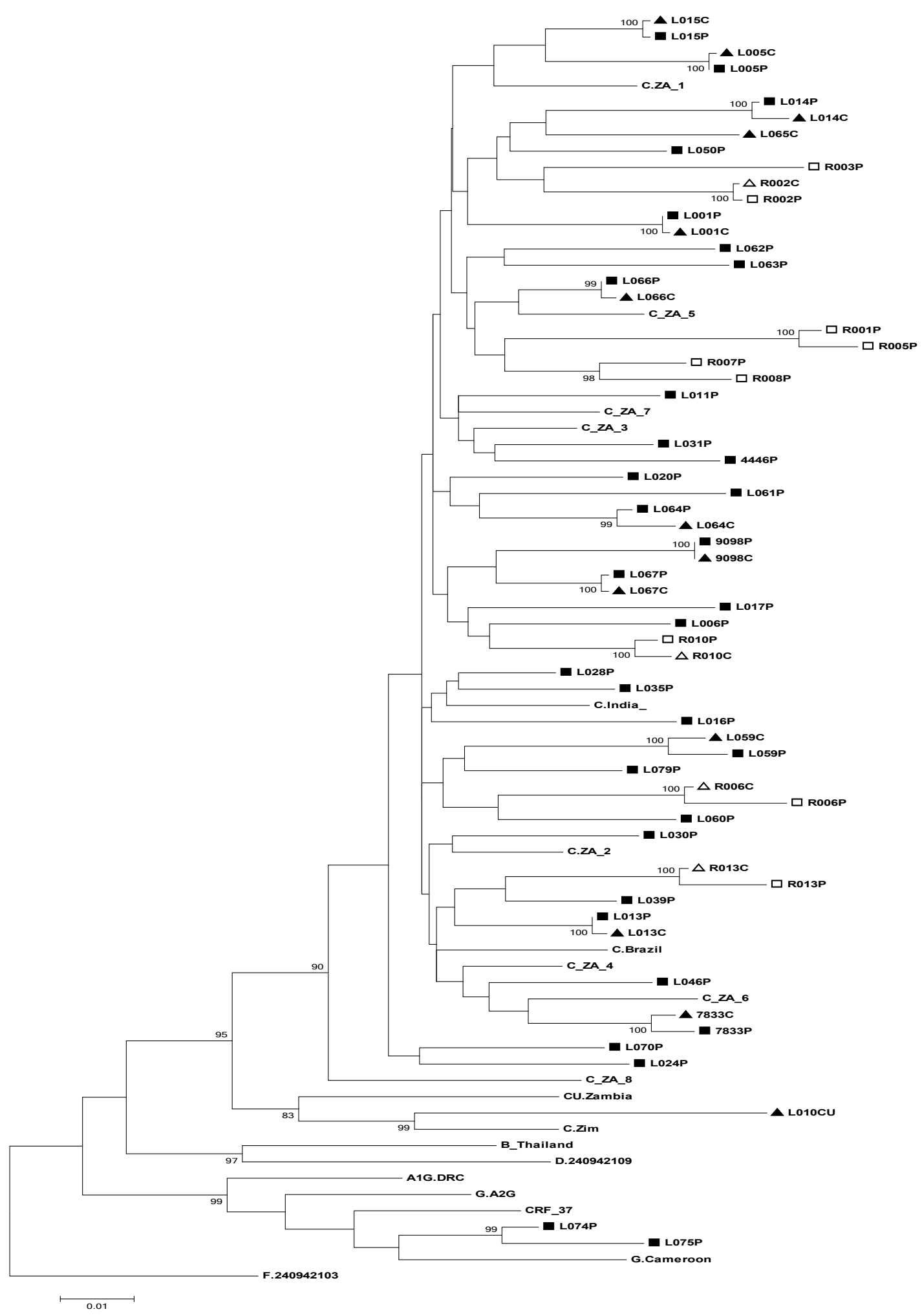

Phylogenetic analysis by Maximum Likelihood method showing sequences from plasma (shaded rectangles $=$ treatment naïve, unshaded rectangles $=$ treatment experienced), PBMCs (shaded rectangles = treatment naïve, unshaded triangles = treatment experienced) and reference sequences. Only sequences covering the whole protease gene and the first 260 amino acids after alignment were used. The percentage of trees in which the associated taxa clustered together is shown next to the branches. The tree is drawn to scale, with branch lengths measured in the number of substitutions per site. The analysis involved 78 nucleotide sequences. All positions containing gaps and missing data were eliminated. There were a total of 1007 positions in the final dataset. The tree is rooted with HIV-1 subtype F reference sequence from the Los Alamos HIV Database as an out-group.

Figure 1: Phylogenetic analysis by Maximum Likelihood method showing sequences from plasma and PBMCs. 


\begin{tabular}{|c|c|c|c|c|c|}
\hline ID & Stanford HIV subtyping & Rega V.3 & SCUEL & $\begin{array}{c}\text { Blast search/ } \\
\text { Phylogenetic analysis }\end{array}$ & Most likely assignment \\
\hline L010 & PR: B, RT: C & Not classified & $\mathrm{U}$ & Subtype $\mathrm{C}$ recombinant & Unclassified \\
\hline L074 & CRF02_AG & CRF37_cpx & CRF37_cpx & AG recombinant & CRF37_cpx \\
\hline L075 & PR: CRF01_AE, RT: G & CRF37_cpx & complex & AG recombinant & CRF37_cpx \\
\hline
\end{tabular}

Table 1: Subtyping results of detected recombinant forms using the HIV-1 pol region.

\begin{tabular}{|c|c|c|c|c|c|c|c|c|}
\hline \multirow{2}{*}{ ID } & \multirow{2}{*}{ Gender } & \multirow{2}{*}{ Age } & \multirow{2}{*}{ CD4 count } & \multirow{2}{*}{ Genotype } & \multicolumn{4}{|c|}{ Resistance associated mutations } \\
\hline & & & & & Plasma PR & PBMC PR & Plasma RT & PBMC RT \\
\hline L001 & M & 48 & 309 & $\mathrm{C}$ & None Detected & None Detected & None Detected & K219Q \\
\hline L006 & $\mathrm{F}$ & 26 & 9 & C & T74S & None Detected & None Detected & None Detected \\
\hline L010 & M & 54 & 350 & Unclassified & None Detected & None Detected & K103N & $\begin{array}{c}\text { M41L } \\
\text { K65R } \\
\text { V751 } \\
\text { F116Y } \\
\text { Q151M } \\
\text { V90I }\end{array}$ \\
\hline L016 & $\mathrm{F}$ & 27 & 345 & $\mathrm{C}$ & None Detected & None Detected & None Detected & $\mathrm{K} 103 \mathrm{~N}$ \\
\hline L017 & $\mathrm{F}$ & 33 & 95 & C & K20M & None Detected & None Detected & None Detected \\
\hline L024 & $M$ & 32 & 25 & $\mathrm{C}$ & $\mathrm{K} 20 \mathrm{M}$ & None Detected & None Detected & None Detected \\
\hline L063 & $\mathrm{F}$ & 43 & 194 & C & None Detected & None Detected & $\mathrm{K} 103 \mathrm{~N}$ & None Detected \\
\hline L067 & $\mathrm{F}$ & 27 & 257 & $\mathrm{C}$ & $\mathrm{T} 74 \mathrm{~S}$ & $\mathrm{~T} 74 \mathrm{~S}$ & None Detected & None Detected \\
\hline
\end{tabular}

Table 2: Resistance testing of plasma and PBMC specimen pairs from treatment-naïve participants harbouring drug resistance associated variants.

\section{PR and RT mutations in plasma and PBMC sample pairs in treatment-naïve individuals}

Paired plasma and PBMC samples from 43 treatment-naïve individuals were tested for HIV drug resistance associated mutations in the $P R$ gene and the first 300 codons of the $R T$ gene. Clear readable sequence data were obtained for 39/43 (91\%) plasma samples compared to $22 / 43$ (51\%) PBMC samples. Table 2 shows participants in which drug resistance associated variants were detected in plasma, PBMCs or both. In the RT gene, HIV drug resistance associated mutations prevalence was $5.1 \%$ in plasma samples compared to $10.3 \%$ when both plasma and PBMC derived sequences were considered despite the low sequencing success rate in PBMC samples. Of the participants with resistance associated mutations, participant L010 was the only one with at least one drug resistance associated mutation in both plasma and PBMC but the mutation profile was discordant (Table 2). There were two participants, L001 and L016, with resistance associated mutations in PBMCs but with no detectable resistance associated mutations in plasma and one participant, L063, with resistance associated mutations detected in plasma only. The K103N mutation which causes high level resistance to first generation NNRTIs Nevirapine (NVP) and Efavirenz (EFV) was the most prevalent, appearing in 3 participants and also associated with the two participants with plasma derived HIV mutations.

No variants with major drug resistance mutations were identified in the $P R$ gene but some $P R$ inhibitor associated minor mutations $\mathrm{K} 20 \mathrm{M}$ and T74S were detected in 4 patients shown in Table 2. Additionally, common polymorphic mutations V82I and L89M not selected by PIs were encountered in up to $56 \%$ of samples and PI inhibitor accessory mutations K20R, T74P and L10M were detected in $22 \%$ of samples. All the PI inhibitor associated mutations detected are unlikely to be due to PI exposure nor would they cause any resistance by themselves.

All participants with plasma and/or PBMC resistance associated mutations at baseline had undetectable viral loads (below 50 copies $/ \mathrm{ml}$ ) after at least six months of HAART except for participant L010 who had a viral load of 147 copies/ml. Patient L001 who had a thymidine analogue mutation-2 (TAM-2), K219Q in the PBMC only, also achieved a full VL suppression at 6 months on a Tenofovir-containing regimen.

\section{PR and RT mutations in plasma and PBMC sample pairs in treatment experienced individuals}

Paired plasma and PBMCs from 10 individuals with virologic failure were also tested for HIV resistance associated mutations in the PR gene and the first 300 codons of the RT gene. PBMC amplification and sequencing was less successful in this group (30\%), compared to the treatment-naïve group (51\%) while $80 \%$ of plasma samples were successfully amplified and sequenced. Of the 3 successfully sequenced PBMC derived variants two sequences, R002 and R013, had resistance associated mutations and no resistance associated mutations were detected from one sequence, R006 (Table 3).The resistance associated mutation profile in PBMC and plasma was the same in R002 but was discordant in the other 2 participants. The M184V mutation, which causes high level resistance to NRTIs lamivudine (3TC) and FTC was the most prevalent mutation in this group appearing in all cases including plasma and PBMC derived variants, followed by the A98G mutation (57\%) and $\mathrm{K} 103 \mathrm{~N}$ and $\mathrm{H} 221 \mathrm{Y}$ both with $43 \%$. The A98G mutation causes resistance to NVP and EFV while the H221Y mutation is an accessory mutation in patients receiving NVP. All treatmentexperienced participants had resistance-associated mutations to both NRTIs and NNRTIs.

\section{Resistance mutations discordance between plasma and PBMC sample pairs}

Intra-host resistant spectra differences in treatment naïve and treatment experienced individuals were compared for 17 individuals with available sequences for both plasma and PBMCs including 14 from treatment-naïve individuals and 3 from treatment-experienced individuals. Resistance spectra differences between plasma and PBMCs in the RT gene were detected in $6(35 \%)$ of these individuals. These participants are shown in Tables 2 and 3 as L001, L010, L016, L063, R002 and R006. In the Protease gene, there were 5 (29.4\%) individuals (L006, L017, L024, R002, and R013) with differences in their resistance spectra between plasma and PBMCs although as previously mentioned 
Page 5 of 9

\begin{tabular}{|c|c|c|c|c|c|c|c|c|c|c|c|}
\hline \multirow{3}{*}{ ID } & \multirow{3}{*}{ Gender } & \multirow{3}{*}{ Age } & \multirow{3}{*}{$\begin{array}{c}\text { Plasma viral } \\
\text { load (copies/ml) }\end{array}$} & \multirow{3}{*}{$\begin{array}{l}\text { CD4 } \\
\text { count }\end{array}$} & \multirow{3}{*}{ Treatment } & \multirow{3}{*}{ Genotype } & \multicolumn{5}{|c|}{ Resistance associated mutations } \\
\hline & & & & & & & \multirow{2}{*}{ Plasma PR } & \multirow{2}{*}{ PBMC PR } & \multicolumn{2}{|c|}{ Plasma RT } & \multirow{2}{*}{ PBMC RT } \\
\hline & & & & & & & & & NRTI & NNRTI & \\
\hline R001 & $\mathrm{F}$ & 36 & 15843 & 304 & $\begin{array}{l}\text { AZT } \\
3 T C \\
\text { NVP }\end{array}$ & $\mathrm{C}$ & L10। & $\begin{array}{l}\text { Could not } \\
\text { amplify }\end{array}$ & $\begin{array}{c}\text { M41L } \\
\text { V75I } \\
\text { M184V } \\
\text { T215Y }\end{array}$ & $\begin{array}{l}\text { A98G } \\
\text { I132L } \\
\text { H221Y }\end{array}$ & $\begin{array}{l}\text { Could not } \\
\text { amplify }\end{array}$ \\
\hline R002 & M & 31 & 69606 & 270 & $\begin{array}{l}\text { TDF } \\
3 T C \\
\text { EFV }\end{array}$ & C & L10V & $\begin{array}{l}\mathrm{L} 10 \mathrm{~V} \\
\mathrm{~T} 74 \mathrm{~S}\end{array}$ & M184V & $\begin{array}{l}\text { L100IV } \\
\text { K103N }\end{array}$ & $\begin{array}{c}\text { M184V } \\
\text { L100I } \\
\text { K103N }\end{array}$ \\
\hline R003 & M & 34 & 5987 & 105 & $\begin{array}{c}\text { TDF } \\
3 T C \\
\mathrm{LPV} / \mathrm{r}\end{array}$ & C & T74S & $\begin{array}{l}\text { Could not } \\
\text { amplify }\end{array}$ & $\begin{array}{l}\text { K70E } \\
\text { M184V }\end{array}$ & $\begin{array}{l}\text { K103N } \\
\text { V108I } \\
\text { K238T }\end{array}$ & $\begin{array}{l}\text { Could not } \\
\text { amplify }\end{array}$ \\
\hline R005 & $\mathrm{F}$ & 29 & 13723 & 168 & $\begin{array}{l}\text { TDF } \\
\text { 3TC } \\
\text { EFV }\end{array}$ & C & L10। & $\begin{array}{l}\text { Could not } \\
\text { amplify }\end{array}$ & $\begin{array}{c}\text { M41L } \\
\text { D67N } \\
\text { V75I } \\
\text { M184V } \\
\text { T215Y }\end{array}$ & $\begin{array}{l}\text { A98G } \\
\text { Y181C } \\
\text { H221Y }\end{array}$ & $\begin{array}{l}\text { Could not } \\
\text { amplify }\end{array}$ \\
\hline R006 & $\mathrm{F}$ & 31 & 140875 & 65 & $\begin{array}{l}\text { TDF } \\
3 T C \\
\text { NVP }\end{array}$ & C & None detected & None detected & $\begin{array}{l}\text { M184V } \\
\text { K101H }\end{array}$ & $\begin{array}{l}\text { Y181C } \\
\text { G190A }\end{array}$ & $\begin{array}{c}\text { None } \\
\text { detected }\end{array}$ \\
\hline R007 & $\mathrm{F}$ & 35 & 109 & 452 & $\begin{array}{l}\text { TDF } \\
3 T C \\
\text { NVP }\end{array}$ & C & T74S & $\begin{array}{l}\text { Could not } \\
\text { amplify }\end{array}$ & M184V & $\begin{array}{l}\text { A98G } \\
\text { K103N }\end{array}$ & $\begin{array}{l}\text { Could not } \\
\text { amplify }\end{array}$ \\
\hline R008 & M & 44 & 9387 & 8 & $\begin{array}{l}\text { TDF } \\
\text { 3TC } \\
\text { EFV }\end{array}$ & C & T74S & $\begin{array}{l}\text { Could not } \\
\text { amplify }\end{array}$ & $\begin{array}{c}\text { K70E } \\
\text { L74V } \\
\text { M184V }\end{array}$ & $\begin{array}{c}\text { A98G } \\
\text { L100I } \\
\text { K103N } \\
\text { H221Y }\end{array}$ & $\begin{array}{l}\text { Could not } \\
\text { amplify }\end{array}$ \\
\hline R013 & $\mathrm{F}$ & 27 & 1800 & 438 & $\begin{array}{l}\text { D4T } \\
\text { 3TC } \\
\text { NVP }\end{array}$ & C & $\begin{array}{l}\text { I54V } \\
\text { L89V }\end{array}$ & None detected & M184V & $\begin{array}{l}\text { K103S } \\
\text { G190A }\end{array}$ & M184V \\
\hline
\end{tabular}

Table 3: Resistance testing of plasma and PBMC specimen pairs from treatment-experienced individuals.

these were minor PR inhibitor associated resistance mutations not selected by PI exposure.

\section{Comparison of plasma and PBMC sample pairs for signature patterns}

Signature pattern analysis on $12 \mathrm{PBMC}$ and 12 plasma sequences done using VESPA showed no differences between the most common amino acid at each position for the PBMC and plasma sequences studied. A threshold of $70 \%$ was considered significant to be an amino acid signature. Although signature markers in PBMCs when compared to plasma were not identified, numerous single nucleotide polymorphisms (SNPs) were detected. Variable amino acid positions in the $P R$ and $R T$ genes are shown in Figures 2 and 3 respectively. We found $21 \%$ of amino acid positions in the PR gene to be variable and $12 \%$ of amino acid positions in the RT gene were variable.

\section{Discussion}

We compared resistance mutations from plasma and PBMC specimen pairs from treatment-naïve and treatment-experienced individuals from South Africa and also analysed the sequences for signature patterns. Although the 5.1\% prevalence figure of plasma HIV drug resistance associated mutations in treatment naïve individuals that we obtained is consistent with figures that have been reported in the region $[14,15]$ the figure was much higher (10.3\%) when drug resistance associated mutations from PBMCs were included. Transmitted drug resistance figures may increase in the near future following the increased roll out of HAART but resistance testing is still not being routinely carried out as part of treatment initiation and monitoring in developing countries because of the associated costs. Prevalence figures of up to $9.3 \%$ resistance-associated mutations in pol sequences from treatment naïve individuals analysed in a South African study have been reported [16].
HIV-1 subtype C, which is the dominant subtype in South Africa, was the most prevalent subtype (94\%). Other subtypes that were identified were HIV-1 subtype CRF37_cpx and an unclassified subtype $\mathrm{C}$ related recombinant form. The CRF 37_cpx subtype has been characterized as a complex strain incorporating subtypes $\mathrm{A}$ and G gene segments and in addition has CRF01_AE and CRF02_AG gene segments [17]. Although the recombinants could not be uniformly assigned by the platforms used, all the platforms used were in agreement on detection of the recombination. The HIV-1 CRF 37_cpx subtype strains were detected in individuals from the central African countries of Congo Brazzaville and the Democratic republic of Congo respectively and have not previously been reported in South Africa.

The Sanger sequencing method is still the gold standard sequencing technique but is typically limited to the detection of mutants that have greater than $20 \%$ prevalence in clinical samples $[18,19]$ and will miss the minority variants in plasma. These minority variants may still be detectable in PBMCs which can harbour earlier variants but their clinical relevance on treatment outcomes is still unclear as some studies have demonstrated that resistance associated minority variants contributed towards therapy failure [19-23] while others have not found a strong association of minor variants with clinical responses [24,25]. In our study the resistant variants detected in proviral DNA did not seem to negatively affect the treatment outcome as viral suppression was achieved after 6 months of treatment. This could be due to the potent regimen that was used, or that the proviral variants are defective and would not be able to be reactivated to contribute a future circulating pool of drug-resistant virus. A patient with a single TAM-2 K219Q also achieved full viral suppression. Detection of other single TAMs such as M41L at baseline has been shown to have no influence on virological outcome on Tenofovir-based regimens [26] while multiple TAM-1 mutations may affect response to Tenofovir $[27,28]$. The resistance associated variants in our group which were dominated by the $\mathrm{K} 103 \mathrm{~N}$ 


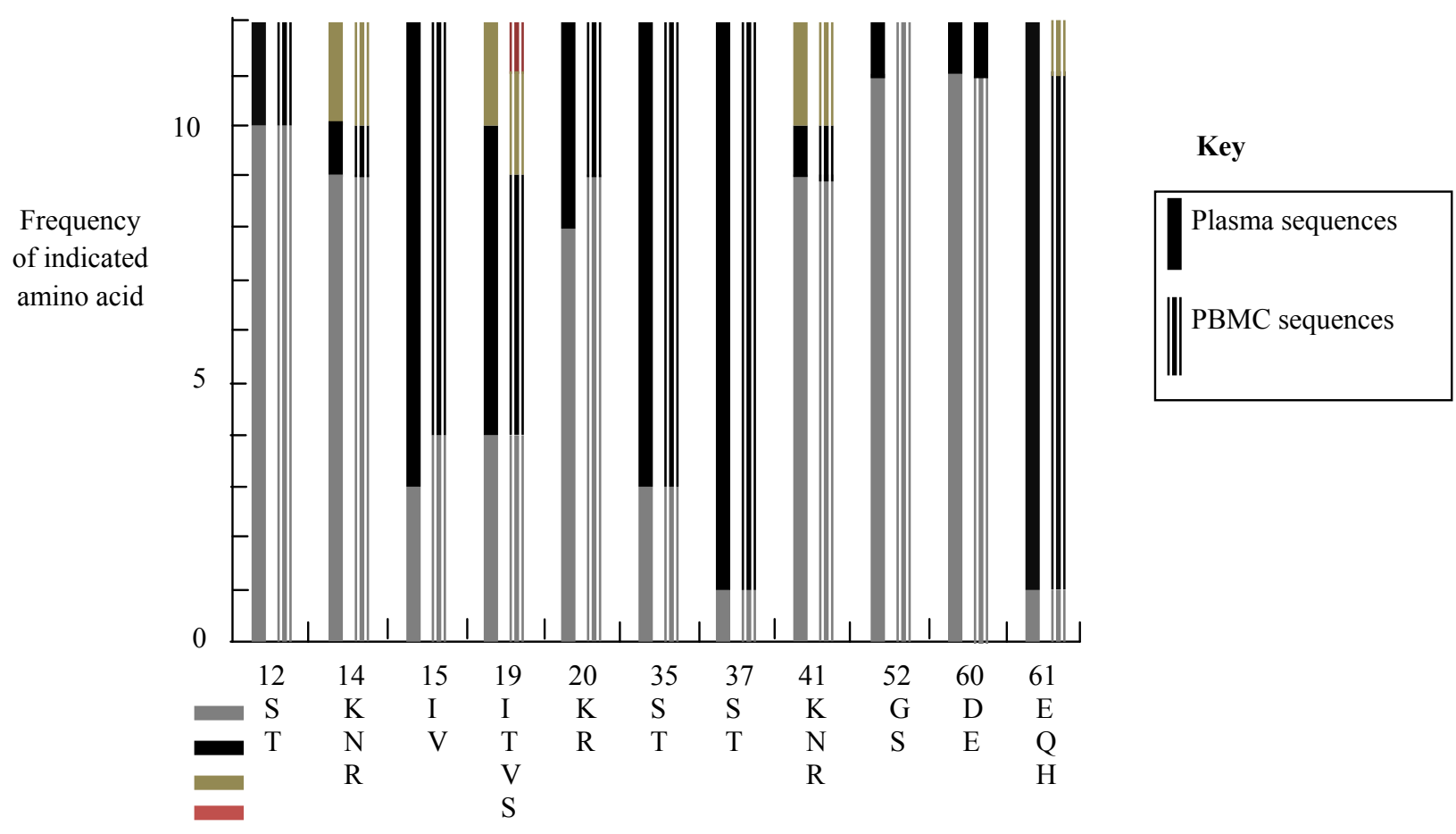

$P R$ gene variable positions and corresponding amino acids
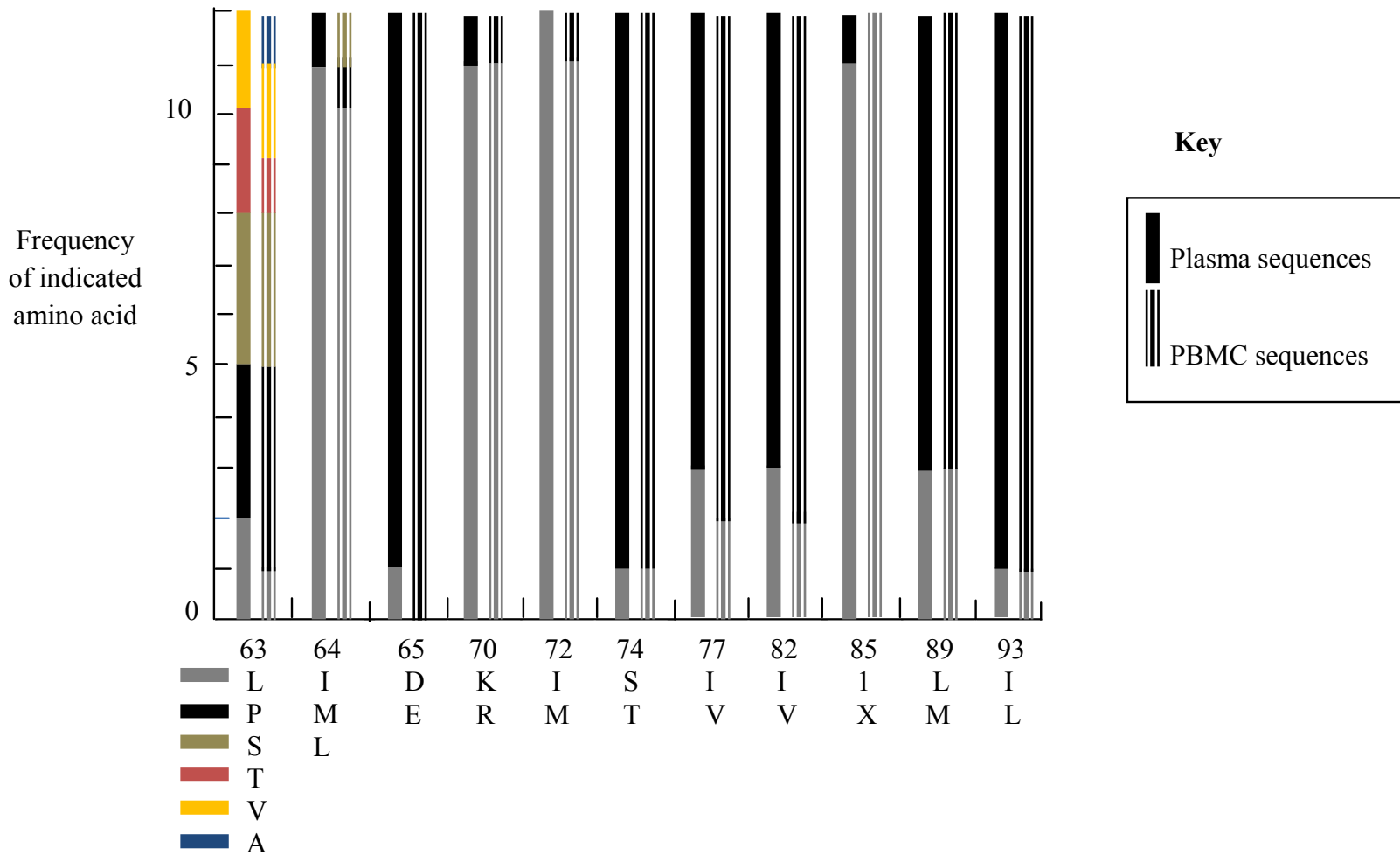

$P R$ gene variable positions and corresponding amino acids

Variable regions in the PR gene for PBMC sequences compared to plasma sequences. VESPA analysis showed no differences between the most common amino acid at each position for the PBMC sequences compared to plasma sequences. There were 19 variable positions (19\% of amino acid positions) in PBMC sequences compared to 21 positions ( $21 \%$ of amino acid positions) that were variable in plasma sequences.

Figure 2: Comparison of the PR gene general variability in plasma and PBMC derived HIV variants. 


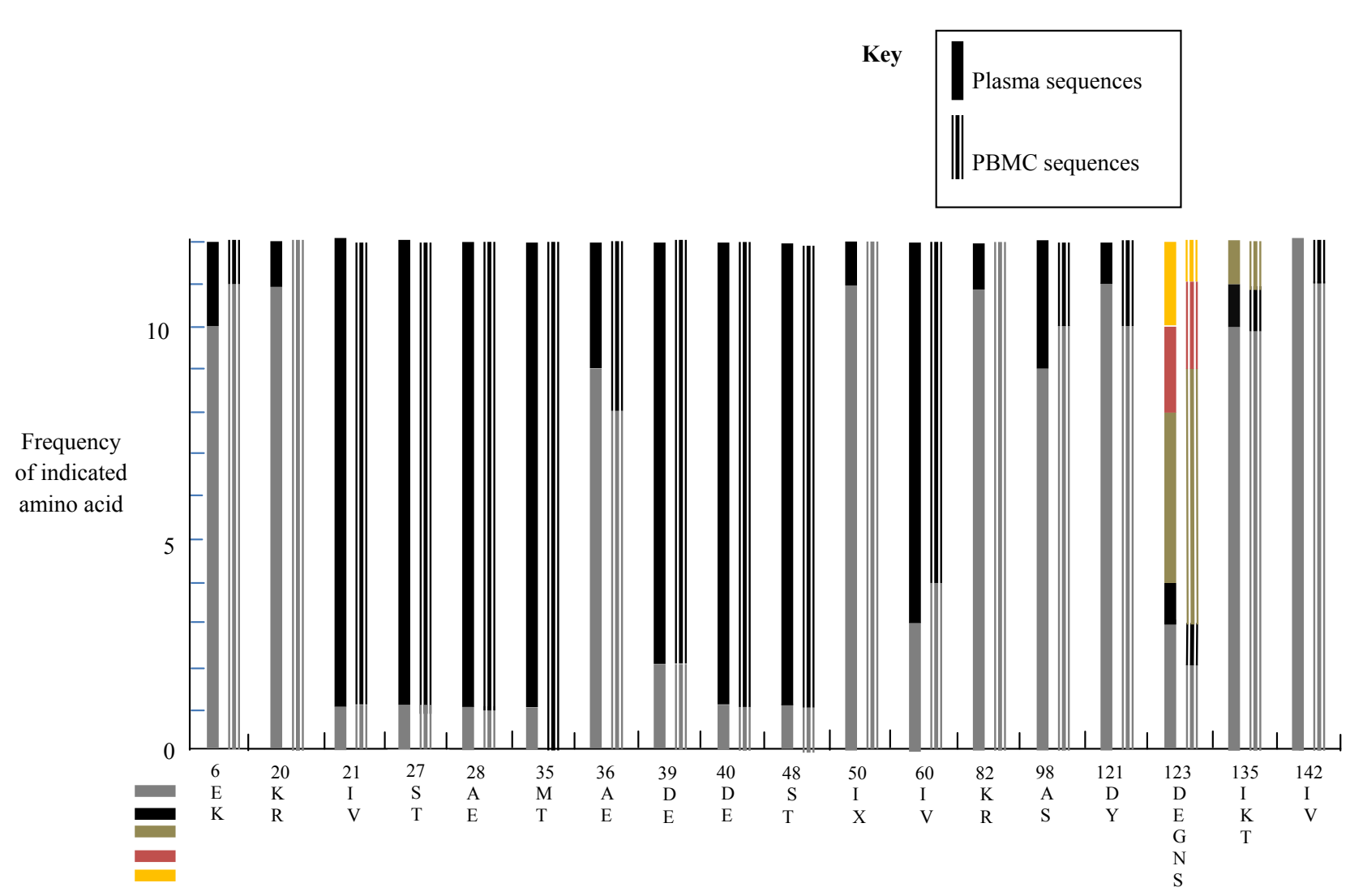

$R T$ gene variable positions and corresponding amino acids

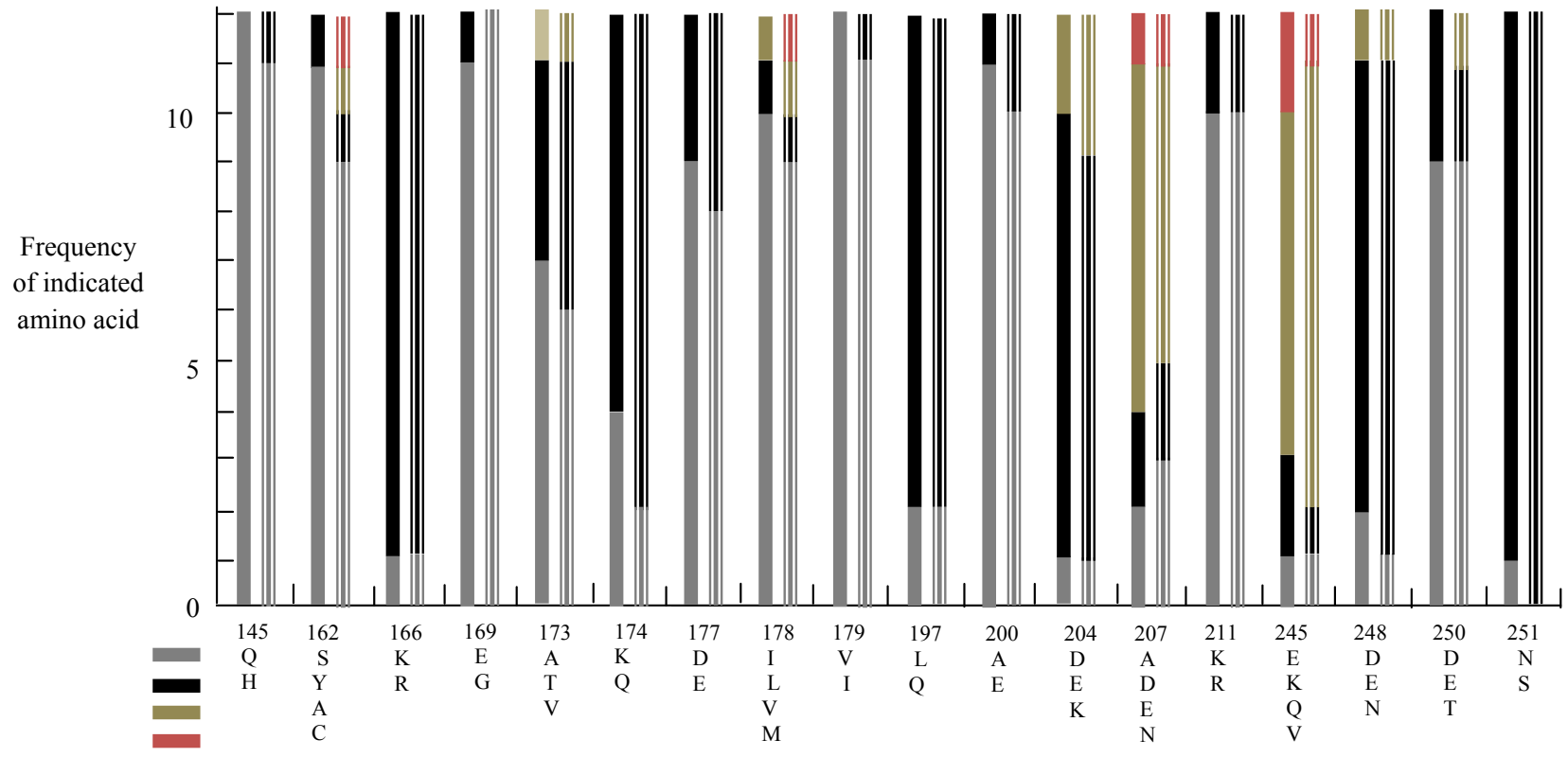

$R T$ gene variable positions and corresponding amino acids

Variable regions in the RT gene for PBMC sequences compared to plasma sequences. VESPA analysis showed no differences between the most common amino acid at each position for the PBMC sequences compared to plasma sequences. There were 30 variable positions (11\% of amino acid positions) in PBMC sequences compared to 33 positions (12\% of amino acid positions) that were variable in plasma sequences.

Figure 3: Comparison of the RT gene general variability in plasma and PBMC derived HIV variants. 
mutation were susceptible to 2 out of the 3 drugs that were used at treatment initiation. The activity of these two drugs, Emtricitabine and Tenofovir, could have led to viral suppression. Sequencing of PBMC variants may also provide information on intra-host virus evolution over the near past and in conjunction with plasma variants detect the emergence of new drug resistant variants in the virus quasi-species population.

There was low PBMC amplification success rate in both treatmentnaive and treatment-experienced patients probably due to the low starting volume of whole blood $(10-15 \mathrm{ml})$ used for PBMC isolation and low $\mathrm{CD} 4 \mathrm{~T}$ cell count. Other PBMC isolation methods have resulted in comparable or higher PBMC amplification and sequencing rate when compared to plasma and these methods include using cell separation tubes [29], collecting blood in citrated tubes [30] and extracting proviral DNA from quantitated PBMC cells [31].

The pol gene is a conserved region of HIV-1 and this was evident in our data as shown by the low intra-host diversity between plasma and PBMC sequences. However there is still some considerable intersubtype diversity in this gene region. In the $P R$ region the $19 \%-21 \%$ variable amino acid positions that we detected are comparable to the 26 out of the 99 protease amino acid positions that have been reported [32]. These polymorphic positions included positions $20,63,82,85$ and 93 which characterize secondary protease mutations in subtype $B$ strains [33]. Position 82 appears on the PR Inhibitor Surveillance Drug Resistance Mutation list [34] but the mutation in our sequences, V82I, is not indicated on the drug resistance associated mutations list. The $11 \%-12 \%$ variable amino acid positions in the $R T$ gene in our data were much lower compared to the reported $23 \%$ polymorphic positions in the first $240 R T$ amino acids [32] and are not associated with resistance mutations except for position 179. This position appears on the NonNucleoside RT Inhibitor Surveillance Drug Resistance Mutation list [34] but the mutation in our sequence V179I is not indicated as a drug resistance associated mutation.

The limitations of this study include the small sample size, lower amplification and sequencing success observed in PBMCs, and that Sanger sequencing only detects variants with a prevalence of $20 \%$ or more. Nonetheless, this study has shown that the sequencing of paired plasma and PBMCs provides additional resistance-associated mutations data which were not detected when only plasma samples are sequenced. We observed a higher prevalence of resistance-associated mutations in treatment-naïve individuals when data from both plasma and PBMCs were considered. These data highlight the presence of proviral HIVDR mutations in HIV infected individuals and longer follow up of patients on treatment may be needed to determine their clinical impact if any, as these variants did not seem to negatively affect treatment outcome at 6 months as viral suppression was achieved. The drug resistance mutations discordance shown between treatment naïve plasma and PBMC sequences may be an indication of inactivity of some of these variants in the short term and in the absence of drug pressure which would otherwise be detected in plasma. To establish what effect resistance-associated HIV variants in proviral DNA might have on treatment outcomes, a bigger sample size as well as more extensive follow up of patients on different types of treatment regimens would be required. The data further showed that differences in resistance spectra may exist between plasma and cellular reservoirs within an individual, highlighting that circulating virus in plasma and provirus in cellular DNA represent distinct compartments that may contribute to the pool of HIV-1 variants that may ultimately emerge in the context of treatment failure.

\section{Acknowledgements}

We acknowledge funding support from the HIV Research Trust, the National Health Laboratory Service Research Trust and the Poliomyelitis Research Foundation.

\section{References}

1. Korber B, Gaschen B, Yusim K, Thakallapally R, Kesmir C, et al. (2001) Evolutionary and immunological implications of contemporary HIV-1 variation. Br Med Bull 58: 19-42.

2. Lauring AS, Andino R (2010) Quasispecies theory and the behavior of RNA viruses. PLoS Pathog 6: e1001005.

3. Ruiz-Jarabo CM, Arias A, Baranowski E, Escarmís C, Domingo E (2000) Memory in viral quasispecies. J Virol 74: 3543-3547.

4. Cohen MS, Chen YQ, McCauley M, Gamble T, Hosseinipour MC, et al. (2011) Prevention of HIV-1 infection with early antiretroviral therapy. N Engl J Med 365: 493-505.

5. Finzi D, Blankson J, Siliciano JD, Margolick JB, Chadwick K, et al. (1999) Latent infection of CD4+ T cells provides a mechanism for lifelong persistence of HIV1 , even in patients on effective combination therapy. Nat Med 5: 512-517.

6. Kantor R, Smeaton L, Vardhanabhuti S, Hudelson SE, Wallis CL, et al. (2015) Pre-Treatment HIV Drug Resistance and HIV-1 Subtype C are independently Associated with Virologic Failure: Results from the Multi-national PEARLS (ACTG A5175) Clinical Trial. Clin Infect Dis 60: 1541-1549.

7. Chargin A, Yin F, Song M, Subramaniam S, Knutson G, et al. (2015) Identification and characterization of HIV-1 latent viral reservoirs in peripheral blood. J Clin Microbiol 53: 60-66.

8. Manasa J, Danaviah S, Pillay S, Padayachee P, Mthiyane H, et al. (2014) An affordable HIV-1 drug resistance monitoring method for resource limited settings. J Vis Exp.

9. Liu TF, Shafer RW (2006) Web resources for HIV type 1 genotypic-resistance test interpretation. Clin Infect Dis 42: 1608-1618.

10. Peña ACP, Faria NR, Imbrechts S, Libin P, Abecasis AB, et al. (2013) Automated subtyping of HIV-1 genetic sequences for clinical and surveillance purposes: Performance evaluation of the new REGA version 3 and seven other tools. Infect Genet Evol 19: 337-348.

11. Kosakovsky Pond SL, Posada D, Stawiski E, Chappey C, Poon AF, et al. (2009) An evolutionary model-based algorithm for accurate phylogenetic breakpoint mapping and subtype prediction in HIV-1. PLoS Comput Biol 5: e1000581.

12. Larkin MA, Blackshields G, Brown NP, Chenna R, McGettigan PA, et al. (2007) Clustal W and Clustal X version 2.0. Bioinformatics 23: 2947-2948.

13. Tamura K, Stecher G, Peterson D, Filipski A, Kumar S (2013) MEGA6: Molecular Evolutionary Genetics Analysis version 6.0. Mol Biol Evol 30: 27252729.

14. Pillay V, Ledwaba J, Hunt G, Rakgotho M, Singh B, et al. (2008) Antiretroviral drug resistance surveillance among drug-naive HIV-1-infected individuals in Gauteng Province, South Africa in 2002 and 2004. Antivir Ther 13: 101-107.

15. Manasa J, Katzenstein D, Cassol S, Newell ML, de Oliveira T; Southern Africa Treatment And Resistance Network (SATuRN) (2012) Primary drug resistance in South Africa: data from 10 years of surveys. AIDS Res Hum Retroviruses 28: $558-565$.

16. Nwobegahay JM, Bessong PO, Masebe TM, Mavhandu LG, Iweriebor BC et al. (2011) Prevalence of antiretroviral drug resistance mutations and HIVsubtypes among newly-diagnosed drug-naive persons visiting a voluntary testing and counselling centre in north eastern South Africa. J Health Popul Nutr 29: 303-309.

17. Powell RL, Zhao J, Konings FA, Tang S, Ewane L, et al. (2007) Circulating recombinant form (CRF) $37 \mathrm{cpx}$ : an old strain in Cameroon composed of diverse, genetically distant lineages of subtypes A and G. AIDS res hum retroviruses 23: 923-933

18. Korn K, Reil H, Walter H, Schmidt B (2003) Quality control trial for human immunodeficiency virus type 1 drug resistance testing using clinical samples reveals problems with detecting minority species and interpretation of test results. J Clin Microbiol 41: 3559-3565.

19. Le T, Chiarella J, Simen BB, Hanczaruk B, Egholm M, et al. (2009) Low- 
abundance HIV drug-resistant viral variants in treatment-experienced persons correlate with historical antiretroviral use. PLoS One 4: e6079.

20. Johnson JA, Li JF, Wei X, Lipscomb J, Irlbeck D, et al. (2008) Minority HIV1 drug resistance mutations are present in antiretroviral treatment-naïve populations and associate with reduced treatment efficacy. PLoS Med 5: e158.

21. Metzner KJ, Giulieri SG, Knoepfel SA, Rauch P, Burgisser P, et al. (2009) Minority quasispecies of drug-resistant HIV-1 that lead to early therapy failure in treatment-naive and -adherent patients. Clin Infect Dis 48: 239-247.

22. Simen BB, Simons JF, Hullsiek KH, Novak RM, Macarthur RD, et al. (2009) Low-abundance drug-resistant viral variants in chronically HIV-infected, antiretroviral treatment-naive patients significantly impact treatment outcomes. J Infect Dis 199: 693-701.

23. Codoñer FM, Pou C, Thielen A, García F, Delgado R, et al. (2011) Added value of deep sequencing relative to population sequencing in heavily pre-treated HIV-1-infected subjects. PLoS One 6: e19461.

24. Peuchant $O$, Thiébaut $R$, Capdepont $S$, Lavignolle-Aurillac $V$, Neau D, et al (2008) Transmission of HIV-1 minority-resistant variants and response to firstline antiretroviral therapy. AIDS 22: 1417-1423.

25. Jakobsen MR, Tolstrup M, Søgaard OS, Jørgensen LB, Gorry PR, et al. (2010) Transmission of HIV-1 drug-resistant variants: prevalence and effect on treatment outcome. Clin Infect Dis 50: 566-573.

26. Pingen M, Nijhuis M, Mudrikova T, van Laarhoven A, Langebeek N, et al. (2015) Infection with the frequently transmitted HIV-1 M41L variant has no influence on selection of tenofovir resistance. J Antimicrob Chemother 70: 573-580.

27. Armstrong KL, Lee TH, Essex M (2009) Replicative capacity differences of thymidine analog resistance mutations in subtype $B$ and $C$ human immunodeficiency virus type 1. J Virol 83: 4051-4059.

28. Crawford KW, Wakabi S, Kibuuka H, Magala F, Keshinro B, et al. (2014) Short Communication: East Meets West: A Description of HIV-1 Drug Resistance Mutation Patterns of Patients Failing First Line Therapy in PEPFAR Clinics from Uganda and Nigeria. AIDS res hum retroviruses, 30: 796-799

29. Diallo K, Murillo WE, de Rivera IL, Albert J, Zhou Z, et al. (2012) Comparison of HIV-1 resistance profiles in plasma RNA versus PBMC DNA in heavily treated patients in Honduras, a resource-limited country. Int $\mathrm{J}$ Mol Epidemiol Genet 3: 56 .

30. Banks L, Gholamin S, White E, Zijenah L, Katzenstein DA (2012) Comparing Peripheral Blood Mononuclear Cell DNA and Circulating Plasma viral RNA po Genotypes of Subtype C HIV-1. J AIDS Clin Res 3: 141-147.

31. Lee GQ, Swenson LC, Poon AF, Martin JN, Hatano H, et al. (2012) Prolonged and substantial discordance in prevalence of raltegravir-resistant HIV-1 in plasma versus PBMC samples revealed by 454 "deep" sequencing. PLoS One 7: e46181.

32. Kantor R, Katzenstein D (2003) Polymorphism in HIV-1 non-subtype B proteas and reverse transcriptase and its potential impact on drug susceptibility and drug resistance evolution. AIDS Rev 5: 25-35.

33. Shafer RW, Kantor R, Gonzales MJ (2000) The Genetic Basis of HIV-1 Resistance to Reverse Transcriptase and Protease Inhibitors. AIDS Rev 2 : 211-228.

34. Bennett DE, Camacho RJ, Otelea D, Kuritzkes DR, Fleury H, et al. (2009) Drug resistance mutations for surveillance of transmitted HIV-1 drug-resistance: 2009 update. PLoS One 4: e4724. 